\title{
MIMO-Based Vehicle Positioning System for Vehicular Networks
}

\author{
Abduladhim Ashtaiwi* \\ Computer Networks Department, College of Information and Technology, University of Tripoli, Libya. \\ * Corresponding author. Email: a.ashtaiwi@it.uot.edu.ly \\ Manuscript submitted September 19, 2014; accepted May 4, 2015. \\ doi: 10.17706/ijcce.2015.4.5.318-326
}

\begin{abstract}
Accurate vehicle positioning is very important factor in many safety applications of vehicle Ad-Hoc networks (VANETs). Two approaches for vehicle positioning have been studied in the literature: global positioning system (GPS) and cooperative positioning system. In this paper we propose MIMO-based vehicle positioning system (MVPS) for vehicular networks. MVPS exploits the spatial channels of multiple input multiple output (MIMO) system. Antenna elements of MIMO system are embodied in proper positions around the vehicle's body. Because of the space separation between antennas elements, signals concurrently transmitted from multiple transmit antennas can easily be separated using their unique fading characteristics. By estimating the distance from each antenna of the transmitting vehicle to each antenna of the receiving vehicle, we can proximate vehicle's position and driving direction. To evaluate the accuracy of MVPS using OPNET Modular we create a simulation model that includes all the vehicular network details for urban area. Comparing the estimated vehicle positioning, obtained from the simulation, to the exact vehicle position, MVPS achieves very high positioning accuracy.
\end{abstract}

Key words: ITS, VANETs, vehicle positioning.

\section{Introduction}

Road crashes continue to be one of the major causes of injury to people in all societies across the world. Intelligent transportation system (ITS) applies information and communication technologies to develop and improve transportation systems of all kinds. One important component in ITS is the development of vehicle Ad-Hoc networks (VANETs), it enables vehicle-to-vehicle (V2V) and vehicle-to-roadside (V2R) communications. The enabled V2V and V2R communications of VANETs opens entirely new possibilities to implement many safety and non-safety applications. The visibility of VANETs applications increased as the US Federal Communication Commission allocated $75 \mathrm{MHz}$ bandwidth of the $5.9 \mathrm{GHz}$ band to dedicated short range communication (DSRC) [1]. Two working groups (WGs), P1609 and 802.11p, have been formed to produce a standard for DSRC. IEEE 802.11p working group, named as wireless access in vehicular environment (WAVE) [2], amended the physical (PHY) and medium access control (MAC) layers of the IEEE 802.11 standard to address the requirements imposed by vehicular networks. The P1609 WG have produced a number of standards numbered as P1609.1 through P1609.4 that focused on issues such as multi-channel operation, cross-layer interfaces, security, and overall architecture [3], [4]. In vehicular networks, the accuracy of vehicular positioning is very crucial factor in most VANETs safety applications. Based on the reviewed literature two classes of vehicular positioning schemes have been proposed: Global 
positioning systems (GPSs) and cooperative positioning systems. In GPS-based positioning systems, the vehicles estimate their positions based on the signals received from four or more GPS satellites. For acceptable positioning accuracy, GPS-based solutions require line-of-sight communications. However, in cooperative positioning systems, vehicles estimate their positions based on signals and positioning information received from surrounding vehicles and from fixed localizing nodes along the roads. In this paper we propose MIMO-based vehicle positioning system (MVPS) for vehicular networks. At scattering environments, signals transmitted from multiple spatially separated antenna elements, undergo unique fading characteristics, known as fading signature. The fading signature is then used at the receiver to separate the concurrently transmitted signals. In MVPS the antenna elements of multiple input multiple output (MIMO) system are embodied in known positions around the vehicle's body. Antennas elements are indexed with code uniquely identify their positions. Signals from each embodied antenna element experience different fading signatures and hence can easily be separated by antennas mounted on the receiving vehicle. By estimating distance from all antenna elements of the transmitting vehicle to all antenna elements of the receiving vehicle, we can estimate the position and driving direction of the surrounding vehicles. To evaluate the accuracy of MVPS using OPNET Modular we create a simulation model for urban area. The created simulation model includes all the vehicular network details such as different roads with different directions, variance vehicular speeds, line-of-sight and non-line-of-sight communications, multipath propagation, etc. Comparing the estimated vehicle positioning, obtained from the simulation, to known position, MVPS achieves very high positioning accuracy.

The remaining of this work is organized as follows: in Section 2 we review therelated works. In Section 3 we give detailed description about the proposed MIMO-based vehicle positioning system. Section 4 describes the simulation environment and the approaches used to evaluate the proposed vehicle positioning system, in this section we also explain the obtained results. In Section 5 we give our final remarks about the proposed positioning system.

\section{Related Works}

Currently, the dominant technology for determining a vehicle's position is the global positioning system. In line-of-sight communication, regular GPS has an average accuracy of approximately 10 meters [5]. Differential GPS (D-GPS) and Assisted GPS (A-GPS) [6] can achieve an average accuracy between 3-7 meters. In [7], a GPS-free positioning algorithm for mobile ad hoc networks was proposed where each node runs a self-positioning algorithm that computes the angles between the one-hop neighbors using the inter-node distance measurements to establish a local coordinate system. Once the local coordinate systems are established, the nodes orient their coordinate system to a common coordinate system such that all nodes' $x$, $y$ coordinates point in the same direction. The authors in [8] proposed a scheme for localizing neighboring vehicles based on radio range measurements. Their goal was to establish an accurate map of the relative positions of all neighboring vehicles. Based on the created map, the proposed scheme uses trilateral for estimating vehicles position. The work in [9] and [10] proposed using anchor nodes or base stations that have knowledge of their own positions. The remaining nodes then communicate with the anchor nodes to determine their locations as they have no other method to estimate their locations. The authors in [11] proposed cooperative positioning scheme based Doppler Effect. However, as line-of-sight condition is not always available in downtown, mountain, and/or canyonareas, GPS and its variance solutions are not always good for vehicular networks where immediate vehicles' positions constantly needed. Similar to GPS-free schemes, our proposed scheme utilizes MIMO technology to estimate position and driving direction of nearby vehicles. Based on the surveyed literature, this is the first time the spatial channels of MIMO technology being proposed to estimate vehicle's position. 


\section{MIMO-Based Vehicle Positioning System (MVPS)}

In this work we assume that each vehicle $V_{u} ; u=\{1,2, \ldots, n\}$ has $k$ MIMO antenna embodied in proper positions around the vehicle's body, as shown in Fig. 1, $n$ is the number of vehicles in the surrounding vicinity of the $V_{u^{t h}}$ vehicle. We use $V_{t x}$ and $V_{r x}$ to refer to the transmitting and receiving vehicles, respectively. In this work we assume that $k=4$, we also assume that each antenna element is indexed with unique code identifies the vehicle and the position of the antennas on the vehicle's body. In this work we denote the antenna's unique code as $a n t_{i}^{V_{u}}, i=\{a, b, c, d\}, u=\{1,2, \ldots ., n\}$ as shown in Fig. 1. By relating to the antennas, embodied on each side, we can map different vehicle's sides (i.e., right, left, front, or back) to different antenna sets as depicted in Table 1. Vehicles in IEEE 802.11p and IEEE 1609 standards periodically broadcast beacon frames at a fixed rate $R$, [12], [13]. Periodically broadcasted beacons enable each vehicle to share its driving information with nearby vehicles. In this work we assume that each vehicle concurrently transmit the broadcasted beacon frame from its $k$ antenna. Nearby vehicles receive the beacon frame using their $k$ receive antennas, i.e., each antenna mounted on the receiving vehicle receives $k$ signals from each transmitting vehicle. As shown in Fig. 1, antenna $a n t_{b}^{V_{r x}}$ of $V_{1}$ is receiving signals from antennas, $a n t_{i}^{V_{t x}}, i=\{a, b, c, d\}$, mounted on Vehicle 2. After receiving the transmitted signal, each receiving antenna on the receiving vehicle, $a n t_{j}^{V_{r x}}, j=\{a, b, c, d\}$, estimates the distance to each of the transmitting antennas, $a n t_{i}^{V_{t x}}, i=\{a, b, c, d\}$, on the transmitting vehicle. Fig. 1 depicts the $k$ estimated distances, $d e\left(a n t_{j}^{V_{r x}}\right.$, ant $\left.t_{i}^{V_{t x}}\right)$ when $j=b$ and $i=\{a, b, c, d\}$. The distance between the $j^{\text {th }}$ receive antenna to the $i^{t h}$ transmit antenna, $d e\left(a n t_{j}^{V_{r x}}, a n t_{i^{t h}}^{V_{t x}}\right)$, is approximated as

$$
\operatorname{de}\left(a n t_{j}^{V_{r x}}, a n t_{i}^{V_{t x}}\right)=\tau_{j, i} C, \quad i=\{a, b, c, d\}, \quad j=\{a, b, c, d\}
$$

where $\tau_{j, i}$ is the propagation delay of the transmitted signal from the ant ${ }_{i} V_{t h}$ antenna mounted on the transmitting vehicle to the $a n t_{j^{t h}}^{V_{r x}}$ antenna mounted on the receiving vehicle, and $C$ is the speed of light. After performingdistance estimation, each antenna on the receiving vehicle would has $k$ approximated distances to $k$ transmitting antennas mounted on the transmitting vehicle as shown in Fig. 1 for antenna $a n t_{b}^{V_{r x}}$.

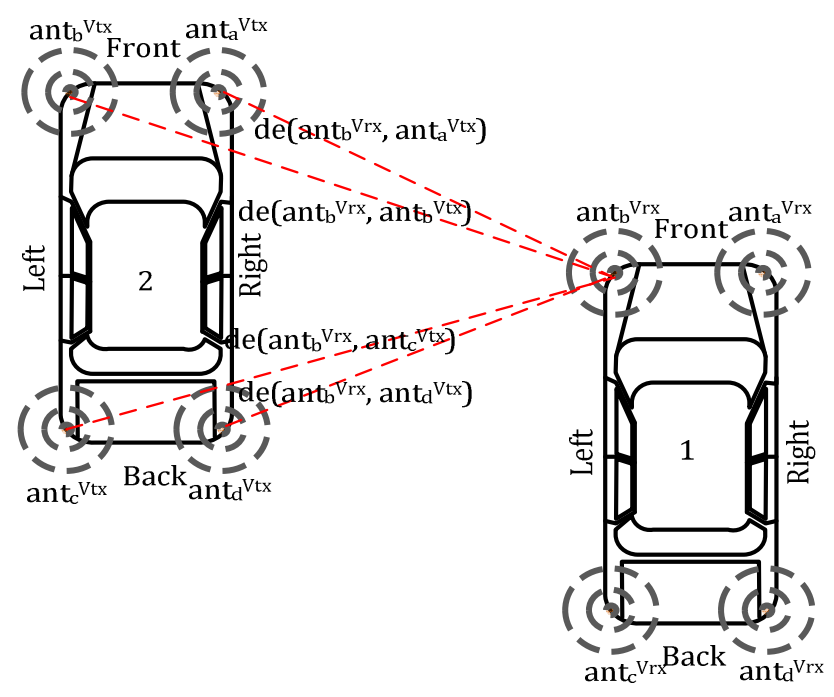

Fig. 1. Antenna position around the vehicle. 
Table 1. Mapping Different Antenna Sets to Different Vehicle's sides

\begin{tabular}{|c|c|}
\hline Antenna Element sets & Vehicle's side \\
\hline$\left\{a n t_{a}^{V_{t x}}, a n t_{b}^{V_{t x}}\right\}$ & Front \\
\hline$\left\{a n t_{b}^{V_{t x}}, a n t_{c}^{V_{t x}}\right\}$ & Left \\
\hline$\left\{a n t_{c}^{V_{t x}}, a n t_{d}^{V_{t x}}\right\}$ & Back \\
\hline$\left\{a n t_{d}^{V_{t x}}, a n t_{a}^{V_{t x}}\right\}$ & Right \\
\hline
\end{tabular}

As shown in the output of Algorithm 1, each antenna, $a n t_{j}^{V_{r x}}, j=\{a, b, c, d\}$, mounted on the receiving vehicle, has $k$ approximated distances to the $k$ transmitting antennas, $a n t_{i}^{V_{t x}}, i=\{a, b, c, d\}$, that mounted on the transmitting vehicle. Given the estimated distance between the $a n t_{j^{t h}}^{V_{r x}}$ to the $a n t_{i^{t h}, i}^{V_{t x}},\{a, b, c, d\}$ antennas, the next step for the receiving antenna is finding its coordinates, ant $t_{j}^{V_{r x}}\left(x_{j}, y_{j}\right), j=\{a, b, c, d\}$ such that the distance from the tried coordinate values to the $a n t_{i}^{V_{t x}}\left(x_{i}, y_{i}\right), i=\{a, b, c, d\}$, is equal to the estimated distances, $d e\left(a n t_{j}^{V_{r x}}, a n t_{i}^{V_{t x}}\right)$. Finding desired coordinates is done by changing $x$ and $y$ values of the receiving antenna.

Algorithm 1: How peer antenna on different vehicles estimate distances.

1. $\forall a n t_{j}^{V_{r x}}, j=\{a, b, c, d\} \mapsto$ mounted on the receiving vehicle

2. loop

3. $\forall a n t_{j}^{V_{t x}}, j=\{a, b, c, d\} \mapsto$ mounted on the transmitting vehicle

4. loop

5. Compute the approximated distance from $a n t_{j}^{V_{r x}}$ to $a n t_{j}^{V_{t x}}$ antenna using Equation (1)

6. Returnde $\left(a n t_{j}^{V_{r x}}, a n t_{i}^{V_{t x}}\right)$ for the $j^{\text {th }}, i^{\text {th }}$ antenna pair

$7 . \quad i++$

8. End loop

9. Returnde $\left(a n t_{j}^{V_{r x}}, a n t_{i}^{V_{t x}}\right), i=\{a, b, c, d\}$ for each $j^{\text {th }}$ antenna

10. $j++$

11. End loop

12. Output:de $\left(a n t_{j}^{V_{r x}}, a n t_{i}^{V_{t x}}\right), i=\{a, b, c, d\}, j=\{a, b, c, d\}$

Equation 2 shows how coordinates of the receiving antenna, $x_{j}$ and $y_{j}$, can be changed until we get a distance (i.e., between the coordinates of the transmitter to the coordinates of the receiver) equal to the estimated distance computed by Equation 1. Equation 2 shows how peer antennas on different vehicles can find coordinates such that the distance from these coordinates to the transmitting antenna coordinates is equal to the estimated distance.

$$
d c\left(a n t_{j}^{V_{r x}}, a n t_{i}^{V_{t x}}\right)=\sqrt{\left(x_{j}-x_{i}\right)^{2}+\left(y_{j}-y_{i}\right)^{2}}, j=\{a, b, c, d\}, i=\{a, b, c, d\}
$$

where $x_{i}, y_{i}, i=\{a, b, c, d\}$ represent the coordinate values of the $i^{\text {th }}$ transmitting antennas and $x_{j}, y_{j}, j=$ $\{a, b, c, d\}$ represent the coordinate values of the $j^{\text {th }}$ receiving antennas. Due to the non-line-of-sight and the presence of multipath the estimated distance, $d e\left(a n t_{j^{t h}}^{V_{r x}}, a n t_{i^{t h}}^{V_{t x}}\right)$, might not be equal to the actual distance. Such error in the estimated distances can make finding coordinates, $a n t_{j}^{V_{r x}}\left(x_{j}, y_{j}\right)$, that returns distances to the transmitting antennas that is equal to the estimated distance, impossible. To overcome this problem we 
proposed Equations 3 and 4 which searches for coordinates that minimizes the difference between the computed distance (i.e., return from the trial of different coordinates) and the estimated distance (i.e., return from simulation).

$$
\begin{aligned}
& \min \left(\sum_{i=0}^{k-1}\left|d e\left(a n t_{j}^{V_{r x}}, a n t_{i}^{V_{t x}}\right)-d c\left(a n t_{j}^{V_{r x}}, a n t_{i}^{V_{t x}}\right)\right|\right), j=\{a, b, c, d\}, i=\{a, b, c, d\} \\
& \text { suchthat } \\
& \left|d e\left(a n t_{j}^{V_{r x}}, a n t_{i}^{V_{t x}}\right)-d c\left(a n t_{j}^{V_{r x}}, a n t_{i}^{V_{t x}}\right)\right| \approx \frac{\sum_{i=0}^{k-1}\left|d e\left(a n t_{j}^{V_{r x}}, a n t_{i}^{V_{t x}}\right)-d c\left(a n t_{j}^{V_{r x}}, a n t_{i}^{V_{t x}}\right)\right|}{k}
\end{aligned}
$$

Algorithm 2 lists the steps of finding the coordinates of the $j^{\text {th }}$ receiving antennas, $a n t_{j}^{V_{r x}}\left(x_{j}, y_{j}\right), j=$ $\{a, b, c, d\}$. As shown in the output of Algorithm 2 we have the antennas coordinates, ant $t_{j}^{V_{r x}}\left(x_{j}, y_{j}\right), j=$ $\{a, b, c, d\}$, of the receiving vehicle. By relating the estimated antennas coordinates of receiving vehicle to the antennas coordinates of transmitting vehicle, we can estimate the position and direction of nearby vehicles.

Algorithm 2: Finding the $a n t_{j}^{V_{t x}}\left(x_{j}, y_{j}\right), j=\{a, b, c, d\}$ of the receiving vehicle

Input:de $\left(a n t_{j}^{V_{r x}}, a n t_{i}^{V_{t x}}\right), i=\{a, b, c, d\}, j=\{a, b, c, d\}$

1. $\forall a n t_{j}^{V_{r x}}, j=\{a, b, c, d\}$ of the receiving vehicle

2. loop

3. Execute equation 2 and 3.

4. return: $a n t_{j}^{V_{t x}}\left(x_{j}, y_{j}\right)$ coordinates of the $j^{\text {th }}$ antenna.

5. $J++$

6. end loop

7. Output: $\operatorname{ant}_{j}^{V_{t x}}\left(x_{j}, y_{j}\right), j=\{a, b, c, d\}$ all $\mathrm{j}$ antennas of the receiving vehicle

\section{Performance Evaluation}

In this section, using simulations we evaluate MVPS scheme. First, we briefly describe the simulation environment, the approaches used to evaluate MVPS and the evaluation metrics used. Then, we present and analyze the simulation results.

\subsection{Simulation Scenario and Parameters}

To evaluate the proposed MIMO-based vehicle positioning system we simulated the scenario shown in Fig. 2 using OPNET Modeler. Vehicles are moving at fixed speed $(S=15 \mathrm{~m} / \mathrm{s})$ in different directions inside an urban area with high rise buildings. In this scenario we consider mix of line-of-sight and non-line-of-sight communications. All vehicles send a small beacon frame $M$ at a fixed rate $R$ using IEEE 802.11p MAC protocol. For simplicity we only evaluate MVPS in Vehicle $V_{1}$. In addition to the receiving vehicle, we considered four other moving vehicles, i.e., 2, 3, 4, and 5; their moving direction and roads are shown in Fig. 2. To model the propagation of the electromagnetic wave between a transmitter and a receiver antenna, we built a simulator that models the wave propagation. In this simulation, the wave propagates through dense graph of dots, as it moves it makes paths of connected dots, the shortest path from the transmitting antenna to the receiving antenna is then returned. We denote the shortest path between the $j^{\text {th }}$ and $i^{\text {th }}$ antenna as $P s_{j, i}, j=\{a, b, c, d\}, i=\{a, b, c, d\}$. Using the returned shortest path, we compute the propagation delay as follows, $P d_{j, i}=\frac{P s_{j, i}}{C}$. To closely model the propagation delay in the selected driving environment, we incorporated the delay spread phenomenon caused by different environments (e.g., highway, rural, 
suburban, and urban areas).

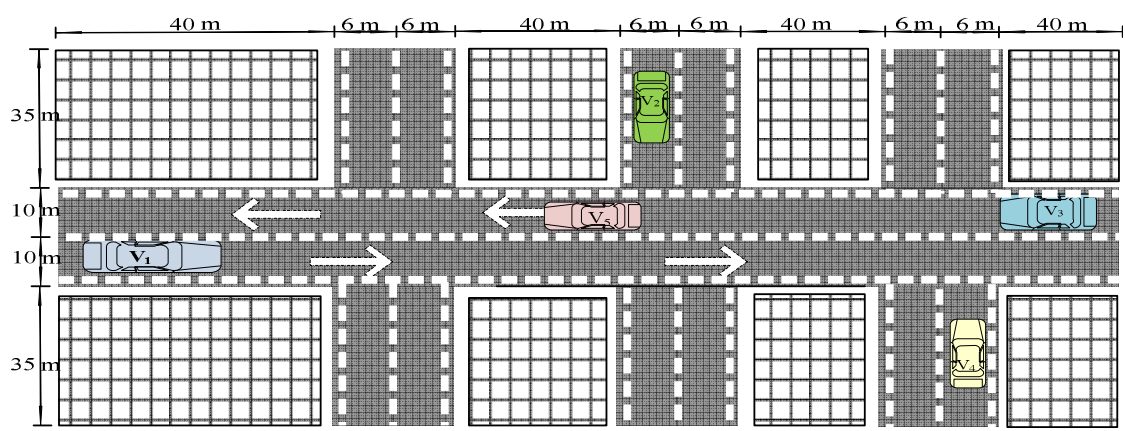

Fig. 2. Modeled driving scenario.

Delay spread is defined as the difference between the time of arrival of the earliest significant multipath component $\left(P d_{j, i}\right.$ in this scenario) and the time of arrival of the latest multipath component. To consider the affect of delay spread, we modeled the propagation delay as Gaussian distribution with $P d_{j, i}$ mean and variance $\sigma^{2}$, i.e., $\mathcal{N}\left(P d_{j, i}, \sigma^{2}\right)$. The variance $\sigma^{2}$ is the wireless channel delay spread which is quantified by Root Mean Square (RMS). Based on RMS measurements done for different areas [14], [15], we selected the values that represent urban areas as shown in Table 2. In this simulation we considered five multipath components $q_{w}, w=\{0,2, \ldots, 4\}$ where $q_{w^{t h}}=\mathcal{N}\left(P d_{j, i}, \sigma^{2}\right)$. Based on the values of the returned propagation delay for five paths, the propagation delay, $\tau_{j, i}$, between the $j^{\text {th }}$ and the $i^{\text {th }}$ antenna is equal to $\frac{\sum_{w=0}^{4}\left(q_{w}\right)}{5}$. Table 2 shows the simulation parameters that we used in this simulation.

Table 2. Simulation Parameters

\begin{tabular}{|l|l|l|l|}
\hline \multicolumn{1}{|c|}{ Parameter } & \multicolumn{1}{|c|}{ Value } & \multicolumn{1}{c|}{ Parameter } & \multicolumn{1}{c|}{ Value } \\
\hline Antenna number $(k)$ & 4 & Number of multipath components $\left(q_{w}\right)$ & 5 \\
\hline Vehicles Speed $(S)$ & $15 \mathrm{~m} / \mathrm{s}$ & Vehicle body length $\left(V_{l}\right)$ & $4 \mathrm{~m}$ \\
\hline Beacon frame size $(M)$ & 40 byte & Vehicle body width $\left(V_{w}\right)$ & $2.4 \mathrm{~m}$ \\
\hline Massage sending rate $(R)$ & $10 \mathrm{~ms}$ & RMS delay spread & $25-40 \mathrm{~ns}$ \\
\hline
\end{tabular}

Vehicle 1 executes MVPS system at each beacon reception. To evaluate the accuracy of the proposed scheme, at each beacon reception we store the exact coordinates of the moving vehicles. We refer to the stored coordinates as $a n t_{i}^{V_{u}}\left(x_{i}^{e x}, y_{i}^{e x}\right), i=\{a, b, c, d\}, u=\{1,2, \ldots, n\}$. The stored coordinates are then compared with the estimated position produced by the MVPS system. To show how much the estimated antenna coordinates (resulted from Algorithm 2) are deviated from the stored antenna position, we computed the shifting distance of each antenna coordinates from its stored coordinates as follows,

$$
\operatorname{shift}\left(\operatorname{ant}_{j}^{V_{u}}\right)=\sqrt{\left(x_{j}-x_{j}^{e x}\right)^{2}+\left(y_{j}-y_{j}^{e x}\right)^{2}}, j=\{a, b, c, d\}
$$

The vehicle's shift (can be referred to as estimation error), $V_{\text {shift, }}$ from its exact position can be expressed as the average of antennas shifts per vehicle. $V_{\text {shift }}$ can be estimated as,

$$
V_{\text {shift }}=\frac{\sum_{\forall j} \operatorname{shift}\left(a n t_{j}^{V u}\right)}{k}, j=\{a, b, c, d\}
$$




\subsection{Simulation Results}

Fig. 3 shows the estimated distances from the antennas mounted on Vehicle 2 to those antennas mounted on Vehicle 1. By observing the distances, shown on Fig. 3, we notice that the distances from antenna ant ${ }_{a}^{V_{1}}$ of Vehicle 1 to the transmitting antennas of Vehicle 2 have different values that represent the relative position of each antenna. Fig. 3 also shows that other antennas of Vehicle 1 have different distance values to the transmitting antenna that represent the relative position of each transmitting antenna to the receiving antenna. By respectively satisfying the distances among antennas as shown on Fig. 3, we can (by drawing lines equal to the estimated distances between antenna peers) approximate the position and driving direction of Vehicle 2. To estimate the transmitting vehicle position and driving direction, each receiving antenna on the receiving vehicle searches for coordinates such that the distance from the tried coordinates to transmitting antennas coordinates are similar or close to the estimated distance. Fig. 4 shows how the receiving antenna chooses the optimum coordinates that satisfy the constraints stated in Equations 3 and 4 .
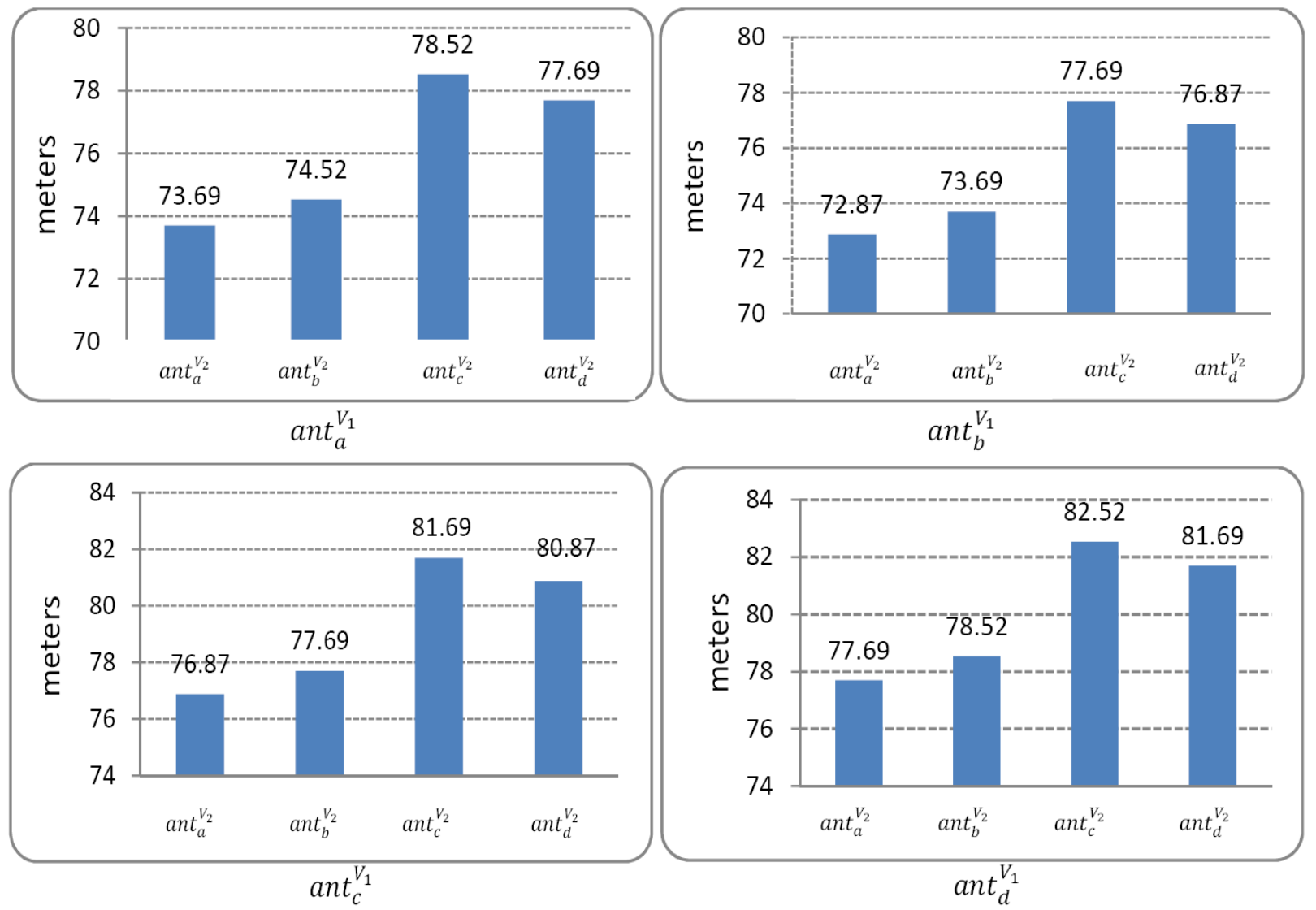

Fig. 3. Estimated distances among antennas mounted on $V_{1}$ to antennas mounted on $V_{2}$.

Fig. 4 shows different values of tried coordinates for antennas $a n t_{d}{ }^{1}$. The shift sum carve, shown on Fig. 4, represents the output of Equation 3, the carve has "V" shape. The smallest value of shift sum represents a valid coordinates point. To satisfy the constraint stated in Equation 4, we need to find the smallest shift sum that has the smallest shift from average, a point where all differences from estimated distances are closely similar. Fig. 4 shows that the coordinate $(44,30)$ satisfy the constraints stated in Equations 3 and 4 . Fig. 5 shows how much the vehicle is shifted from its actual position that is stored during beacon reception. Vehicle's shift (can be referred to as position estimation error) is estimated by Equations 5 and 6. By referring to the simulated driving environment, shown in Fig. 2, we observe that Vehicles 2 and 4 have non line-of-site communications. While Vehicles 3 and 5 have line-of-site communications. By comparing the 
obtained results we notice that the vehicle's shift is less for those with line-of-site communication vehicles, i.e., Vehicles 3 and 5. We can also notice that Vehicle 5 has less vehicle's shift than Vehicle 3, that is because Vehicle 3 has closer distance compared to Vehicle 5. While vehicles of non line-of-site communication such as Vehicles 2 and 4 showed higher vehicle's shift compared to vehicles communicate with line-of-site.

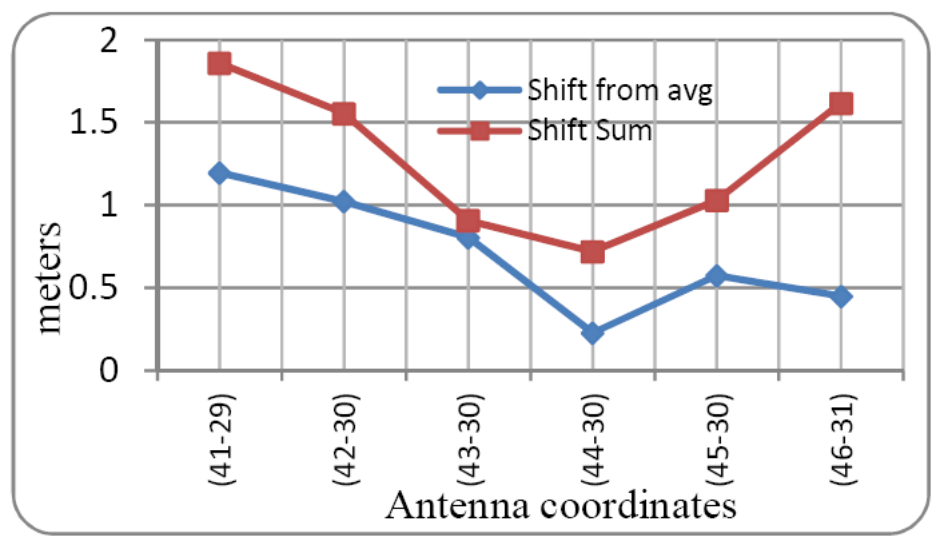

Fig. 4. Finding antenna coordinates.

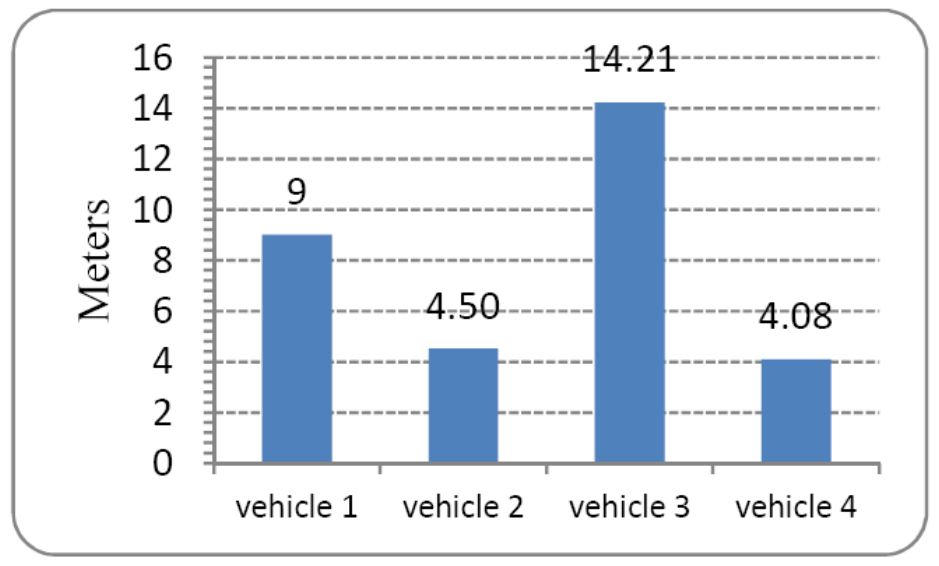

Fig. 5. Vehicle estimation error.

\section{Conclusion}

Knowing vehicle positioning can help implement many effective safety applications in VANETs. In this paper we proposed MIMO-based Vehicle Positioning System (MVPS) for vehicular networks. MVPS exploits the created spatial channels, via using Multiple Input Multiple Output (MIMO) system, to provide vehicle positioning for VANETs. In MVPS antenna element of MIMO are embodied in known position around the vehicle. Antenna elements are indexed with unique code that identifies the vehicle and the positions on the vehicle's body. By estimating distance between peer antenna elements mounted on different vehicles, we can estimate the position and driving direction of the surrounding vehicles. The obtained results from the simulation showed that MVPS achieves very high vehicle positioning accuracy. Vehicles with line-of-sight communication showed better accuracy compared with non-line-of-sight communications. Close vehicles with line-of-sight achieved the highest vehicle positioning accuracy.

\section{References}

[1] SAE International. Dedicated Short Range Communications (DSRC) Message Set Dictionary, SAE J2735. 
From http://www.sae.org/standardsdev/dsrc/

[2] Xiang, W. D., Richardson, P., \& Guo, J. H. (2006). Introduction and preliminary experimental results of wireless access for vehicular environments (WAVE) systems. Proceedings of 2006 Third Annual International Conference Mobile and Ubiquitous Systems: Networking \& Services: Vol. 99 (pp. 1-8).

[3] Task Group P. (2007). IEEE 1609.3-2007 WAVE Networking Services. IEEE Computer Society.

[4] Task Group P. (2007). IEEE 1609.4-2007 WAVE Multi-Channel Operation. IEEE Computer Society.

[5] Drane, C., \& Rizos, C. (1997). Positioning Systems in Intelligent Transportation Systems. London: Artech House. Vol. 99, pp. 230-231.

[6] Djuknic, G. M., \& Richton, R. E. (Feb. 2001). Geolocation and assisted GPS. Computer, 34(2), 123-125.

[7] Capkun, S., Hamdi, M., \& Hubaux, J. (January 2001). GPS-free positioning in mobile Ad-Hoc networks. Proceedings of Hawaii Int. Conf. on System Sciences.

[8] Kukshya, V., Krishnan, H., \& Kellum, C. (September 2005). Design of a system solution for relative positioning of vehicles using vehicle-to-vehicle radio communications during GPS outages. Proceedings of IEEE Vehicular Technology Conference (pp. 1313-1317).

[9] Lazos, L., Poovendran, R., \& Capkun, S. (April 2005). ROPE: Robust position estimation in wireless sensor networks. Proceedings of the Fourth international symposium on Information Processing in Sensor Networks (pp. 324-331).

[10] Niculescu, D., \& Nath, B. (November 2001). Ad-Hoc positioning system (APS). Proceedings of GLOBECOMM: Vol. 5 (pp. 2926-2931).

[11] Nima, A., Asghar, T., \& Andrew, G. (2009). A cooperative positioning enhancement method based on Doppler effect for vehicular networks with GPS availability. VT-01264.

[12] IEEE Standard. (July 2010). 802.11 Working Group, Wireless LAN Medium Access Control (MAC) and Physical Layer (PHY) Specifications, Amend. 6: Wireless Access in Vehicular Environments.

[13] IEEE Standard. (Feb. 2011). 1609 Working Group, IEEE Standard for Wireless Access in Vehicular Environments (WAVE). Multi-Channel Operation.

[14] Zhao, X. W., Jarmo, K., Pertti, V., \& Kari, S. (April 2002). Propagation characteristics for wideband outdoor mobile communications at $5.3 \mathrm{GHz}$. IEEE Journal on Selected Areas in Communications, 20(3), 507-514.

[15] Christoph, F., Johan, K., Alexander, P., Thomas, Z., \& Nicolai, C., (July 2011). Vehicular channel characterization and its implications for wireless system design and performance. Proceedings of IEEE, 99(7), 1189-1212.

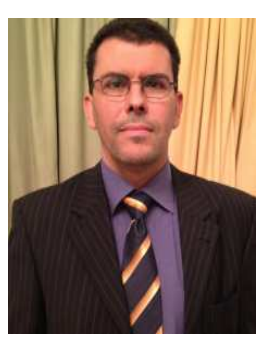

Abduladhim Ashtaiwi was born in Tripoli, Libya, he received his M.Sc and Ph.D. degrees from the Department of Electrical and Computer Engineering (ECE) of Queen's University, Canada in 2004 and 2009, respectively. He worked as a research associate at Queen's University, ECE Department, from Jan. to July 2009. He worked as a systems design consultant with National Economic Development Board (NEDB). He is currently a faculty member and the head of the Computer Networks Department in the College of Information and Technology, University of Tripoli, Tripoli, Libya. His areas of interest include 4G and beyond wireless network architectures, multiple-input multiple-output (MIMO) systems, wireless medium access control techniques, QoS routing and resource reservation. Dr. Ashtaiwi is currently deeply evolved in road safety and efficiency research area. 